\title{
Gaps in continuity of care at the interface between primary care and specialized care: general practitioners' experiences and expectations
}

\author{
This article was published in the following Dove Press journal: \\ International Journal of General Medicine \\ II November 201। \\ Number of times this article has been viewed
}

\section{Ryan Tandjung \\ Thomas Rosemann \\ Nina Badertscher \\ Institute of General Practice and Health Services Research, University of Zurich, Switzerland}

Introduction: Discontinuity of care at the interface between inpatient and outpatient management can lead to increased morbidity and mortality. Appropriate communication and flow of information is indispensable to ensure continuity of care. Consequently, the aim of this study was to assess general practitioners' (GPs) experiences of cooperation with a university hospital, as well as their needs and obvious barriers regarding an optimized information flow. Methods: A qualitative study was performed. In March 2011, 300 GPs from the Zurich Canton were invited to participate in two focus group meetings. Based on a review of the literature, an interview guide was created addressing two main issues. In the first part, experiences and barriers regarding cooperation with the university were explored. In the second part, needs and suggestions to improve cooperation were addressed.

Results: Fifteen GPs participated in two focus groups. GPs complained that they have often not been adequately informed about ongoing treatments or appointments for their patients. GPs feel responsible for the continuity of care and wish to be more involved, especially in longterm treatment decisions or at the end of life. By not involving them, they stated, important information concerning patients' medical history and social setting was not taken into account. Improvements are also required at discharge: GPs often do not receive important information about treatments in the hospital and further requirements within a reasonable time.

Conclusion: Exchange of information between the hospital and the GP at admission and discharge is essential. However, at present, involvement during hospitalization of the patient is lacking. This includes the exchange of information after an unexpected clinical procedure and input from GPs when difficult clinical decisions are made, such as at the beginning or termination of long-term therapies.

Keywords: referral, general practitioners, hospital, cooperation, handover, communication

\section{Introduction}

Discontinuity of care on referral to and discharge from hospital is associated with a high risk of adverse events. After discharge, for example, a changed drug therapy is associated with increased emergency department visits, readmissions, and even disabilities or death. ${ }^{1-3}$ A Canadian study showed that $19 \%$ of patients suffered an adverse event after discharge, $70 \%$ of which were either preventable or ameliorable. A major contributing factor to these adverse events was the lack of communication between the hospital physician and the patient's general practitioner (GP). ${ }^{4}$
Correspondence: Ryan Tandjung Institute of General Practice and Health Services Research, University of Zurich, Pestalozzistrasse 24, 8091 Zurich, Switzerland Tel +4l 442559855 Fax +4I 442559097 Email ryan.tandjung@usz.ch 
GPs are responsible for outpatient management. By the time a hospitalization is necessary, handover to a hospitalist or a hospital-affiliated specialist is common. Good communication in these transitions of care is crucial and may reduce adverse events, both in- and outside the hospital. ${ }^{2,4-8} \mathrm{At}$ admission, the GP can provide the hospital physicians with essential information such as patient history, prior results, and patient background. At discharge, timely information from the hospital should reach the GP, containing diagnosis, known and pending results, the actual medication, and the most important next steps. Based on these premises, guidance for a standard in communication between GPs and hospital physicians has been established., ${ }^{9,10}$

In Switzerland, the role of the GP as a "gatekeeper" is not generally defined. A hospitalization is not mandatorily initiated by the patient's GP; the patient can also be admitted by any GP on call, or any specialist, or even by the patient themself (through an emergency department visit). The Canton of Zurich is the biggest Canton in Switzerland and has around 1.3 million habitants; the capital is the City of Zurich, the biggest Swiss city. The Canton of Zurich has several public and privately funded hospitals, including University Hospital Zurich, where specialized care is provided. The university hospital in Zurich has 42 divisions and institutes and a capacity of 900 inpatient beds.

This setting reflects the typical structure in Switzerland where five university hospitals are spread over the country, serving almost 8 million habitants. These structures of inpatient and outpatient management are comparable to several countries in Western Europe, such as Germany and Austria.

The aim of this study was to assess GPs' experiences of cooperation with a university hospital as well as their needs and obvious barriers regarding an optimized information flow.

\section{Methods}

The authors sought to understand the individual experiences and requests of the GPs concerning cooperation with a university hospital; therefore, a qualitative approach with focus group interviews seemed to be the most appropriate approach. GPs working in the greater area of Zurich were invited to participate in the study. Three hundred GPs (of about 1500 eligible GPs working in the Canton of Zurich) were selected at random and invited by letter and reminder telephone calls. Eventually, 15 GPs participated in two focus group interviews, which were held in March 2011. GPs received SFr200 for participation. The interviews were led by two trained interviewers from the Institute of General Practice, Zurich, Switzerland.

\section{Interview guide}

An interview guide was developed based on a review of the literature, which searched for studies focusing on handover between GPs and hospitals. The interview guide was piloted with two GPs affiliated to the Institute of General Practice who did not participate in the focus group interviews. The interview guide was divided into two parts, both concerning proceedings in patient care. In the first part, experiences and barriers regarding cooperation with the university were explored. In the second part, needs and suggestions to improve cooperation were addressed. In the second part, specific interventions to ameliorate cooperation were also discussed, for example, an online referral form, a directly accessible online schedule, or further efforts such as a newsletter from the hospital or visits from representatives of the hospital.

\section{Analysis}

The interviews were digitally recorded. Detailed protocols were written in German and the analysis was done by two different researchers with ATLAS.ti (v 5.0; ATLAS.ti Scientific Software Development $\mathrm{GmbH}$, Berlin, Germany) software. ${ }^{11}$ To analyze the data, a qualitative approach according to Mayring ${ }^{12}$ was used, specifically, summative content analysis. A categorizing system was established based on the interview guide, with minor adaption during analysis. The interviews were independently read and categorized. Categories are listed in Table 1.

\section{Results}

\section{Participants}

Fifteen GPs participated in the study. They were divided into two groups of six and nine GPs. The average age of the participants was 56 years (range, 42-65 years); on average they had been in private practice for 21 years (range, 11-30 years). Five of the fifteen GPs were female. Of the 15 GPs, 13 worked in the City of Zurich, and two in rural areas. Only two GPs were working in a single-handed practice, and 13 were in a practice with at least two doctors. Seven of the GPs had digital medical records, while eight GPs were working with paper-based records.

Table I Categorizing system for the interviews

Negative experiences

Positive experiences

Comparison of experiences with other hospitals/Benchmark

Expectations for future cooperation 


\section{Proceedings in patient care}

As previously discussed, the interview guide contained two parts. The first part concerned experiences from past cooperation. In this part, comparisons with other hospitals as well as differences between clinics at the university hospital were discussed. The second part addressed the need for future cooperation with the university hospital.

\section{General aspects}

Several GPs stated that they saw large hospitals such as the university hospital as kind of a "black box" and highlighted the importance of a minimum standard of communication.

My perception of the university hospital is the perception of a black box: the patient enters, sometimes he leaves; for the GP no influence or information lies in between. (GP3, $\mathrm{m}, 64$ years)

\section{Admission}

Regarding admission of a patient, two major points were raised by the GPs. Most GPs stated that, after an unexpected admission, which was not initiated by the GP, they expected to be informed within a day about the hospitalization of the patient.

In the context of planned admissions, the second issue mentioned referred to communication problems with written referral letters: while an emergency admission is usually managed on the phone, an elective hospitalization or diagnostic procedure usually requires a written letter from the GP, normally sent by post or facsimile. While the patient receives a notification about the scheduled intervention, the doctor who arranged for this procedure is frequently not informed until the intervention has been performed. Different methods of communication, including digitally by email, through an online form, or using a directly accessible online schedule, were discussed. Most GPs were quite flexible about the method of communication. They clearly stated that it is not how communication takes place that is important, but the fact that it takes place at all.
The lack of different possibilities is not the problem, but the fact that after the referring letter is submitted, a referring doctor won't hear anything anymore. (GP1, m, 55 years)

\section{During hospitalization}

During hospitalization, again the lack of information due to noncommunication was mentioned as the main reason for dissatisfaction with the care provided in the university hospital. If standard procedures are performed and no complications occur, it may be appropriate to dispense information at the end of the stay. However, if problems or unexpected referrals occur within the hospital, especially referrals to the intensive care unit, the GPs stated that information is needed. In addition to the fact that the GP themself is interested in these referrals, many GPs stated that during the hospital stay they are often confronted with patients' relatives, who also seek information.

I want to be informed if a patient has to be transferred to the intensive care unit: after all, relatives are often contacting me in case a complication occurred in the hospital. (GP9, $\mathrm{m}, 63$ years)

A list of minimal levels of communication during a possible clinical procedure is shown in Table 2.

Some GPs wanted to be more involved in decisions of a long-term nature, such as the start of dialysis in a geriatric patient, or the need for further diagnostics in multimorbid patients.

In multimorbid, complex patients, where for example dementia plays an important role, I would really appreciate it if we - altogether in the team - could discuss where to put priorities. (GP3, m, 64 years)

In these situations, GPs regarded themselves not only as information receivers but also as part of the medical team. They stated that they could contribute to difficult decisions such as end-of-life situations, which frequently involve patients' perspectives on individual ethical values. Due to

Table 2 General practitioners' (GPs) expected minimal standard for communication between hospitals/hospital physicians and GPs

\begin{tabular}{llc}
\hline Admission & Information to the GP during hospitalization & Discharge \\
\hline $\begin{array}{l}\text { - Notification copy is sent to both } \\
\text { the patient and the GP }\end{array}$ & - After an emergency admission & - Short letter is sent to the GP's office the day \\
after the patient is discharged & - Discharge letter is sent to the admitting \\
doctor too (if different from the GP) & - When complications, unexpected events occur & - Referral to another clinic (within hospital or to \\
another institution) & - When long-term therapeutic decisions have to be taken \\
& - Death
\end{tabular}


the often long-lasting patient-physician relationship, GPs often have detailed information about these aspects. They complained that their colleagues at the hospital are not aware of that and therefore important or at least helpful information is not taken into account when these kinds of decisions are made in the hospital.

In unexpected findings or in an unexpected severe course or in decisions where the psycho-social background is important, it is reasonable to involve the GP. (GP11, m, 53 years)

A resident, who does not know a patient and only has a short referral letter in front of him, should have a different approach: "What further information do I need to correctly allocate further diagnostics and therapy?" If he asks himself this question he will automatically contact the patient's GP. (GP1, m, 55 years)

\section{Discharge}

Discharge papers were a major issue for all participating GPs. Timely information about the hospitalization is most important, especially when a prompt follow-up at the GP's office is indicated. There was a clear consensus that this information should get to the GP not later than the day after discharge. Short forms of discharge papers should at least contain the actual diagnoses, possible complications, the actual medication, and the immediate following procedures.

The way the discharge papers should reach the GP was also an important issue. The main options were paper-based versus digital. GPs statements reflected no uniform opinion: the GPs who already worked with electronic patient files explicitly asked for a digital version of the discharge letter. GPs mostly working with paper-based records and not regularly using information technology systems in their practice voted for a paper-based discharge letter.

\section{Benchmarks}

All GPs had experiences with several different hospitals or different departments within the university hospital. All stated that there is a wide variation in the quality as well as the quantity of communication. Positive experiences were used as a benchmark in their statements. All GPs complained that the university hospital has obvious deficits in communication compared with other hospitals. Some procedures reflecting good hospital-GP communication were mentioned:

There are occasional phone calls from residents from the university hospital, sometimes at discharge, sometimes when a patient dies. I appreciate these phone calls, but other hospitals work more reliably. (GP3, m, 64 years)

In other hospitals communication between hospital physicians and GPs works at a much lower threshold. (GP1, m, 55 years)

To assess possible interventions to improve the communication and information flow at the interface, specific innovations were presented, such as newsletters from the hospital to the GPs or visits from hospital representatives to a private practice. All these innovations were assessed very skeptically by most of the GPs. They all stated that the most important part of good cooperation is the provision of good services in patient care.

A good relationship between a GP and a hospital is defined by the hospital's service; if the provision of service is not working, all other efforts are useless. (GP11, m, 53 years)

\section{Discussion}

This study revealed that in many situations GPs were not adequately or not at all informed about ongoing treatments or appointments for their patients from the corresponding university hospital. The lack of adequate and timely communication between hospital physicians and GPs led to dissatisfaction in the group of GPs but may also have a negative impact on treatment decisions in the hospital, when important information about patients' background, setting, and ethical values are needed.

\section{Participants}

Fifteen GPs from Zurich and the region of Zurich participated in the study. Compared with the Swiss average of GPs in private practice, the sample was about the same age. ${ }^{13}$ Seven out of $15(46.7 \%)$ participants worked with electronic medical records, as compared with the last available data in Switzerland, which showed $11.7 \%$ of GPs having electronic medical records in 2007. ${ }^{14}$ Most of the GPs in the present study were working in a practice with at least two doctors. The traditional practice form in Switzerland is a single-handed practice, but, unfortunately, no actual data about the distribution of different practice forms exist.

\section{Admission}

When a GP refers a patient for a routine treatment they would like to know when the appointment is scheduled and to immediately receive the results of the appointment. A simple copy (by postal letter, email, or facsimile) of the scheduled 
procedure(s) - which will be sent to the patient in any case would be the easiest solution. Many hospitals have already established this method of communication.

\section{During hospitalization}

GPs clearly stated that they want to be more involved in the treatment process; they see themselves as very important sources of information. GPs often care for their patients for several decades and have knowledge of both their social and medical background. GPs see themselves as constant caregivers and, during a hospitalization, as part of the care team. They are willing to contribute to difficult decisions in a clinical course, such as starting a long-term therapy on dialysis, or reducing a therapy in the final stages of palliative care.

GPs expect that hospital physicians should inform them in case of an unexpected clinical procedure, such as a referral to intensive care due to a complication. As mentioned, GPs see themselves as part of the care team, and many relatives often rely on the patient's GP and call on the GP when looking for further information about the patient's hospitalization.

These two points show the importance of thorough communication between hospital physicians and GPs. This communication is not just seen as a transfer of information from the hospital to the GP, but also as an interaction between two medical teams, both responsible for the medical treatment of their patient, described by Lee and Garvin as "moving from information transfer to information exchange." 15 Patients rely on the GP being well informed about their clinical course, and both patients and GPs expect good communication between hospital physicians and GPs. ${ }^{16,17}$ In a meta-analysis studying interventions to ameliorate coordination between GPs and outpatient specialists, Foy et al showed that studies aimed at enhancing the quality of information exchange had larger effects on patient outcomes than those that did not. ${ }^{18}$

\section{Discharge}

Expectations regarding the content of discharge papers, especially in terms of diagnosis, actual medication, complications, and immediate proceedings, are consistent with other study results ${ }^{7,16,19}$ and existing guidance. ${ }^{9,10}$ Concerning the transmission of discharge papers, different expectations were voiced. Some GPs - especially those working with full electronic medical records - request only digital discharge papers; some like to have paper-based discharge letters. GPs should be able to state their preference to the hospital and receive further reports in the form they request. This preference might change in future, especially when electronic medical records become more common. ${ }^{14}$ The correlation between communication and clinical outcomes is ambiguous. While Hess et $\mathrm{al}^{20}$ showed that a verbal report by telephone added to a written report in prolonged respiratory failure could reduce readmission rate and save costs, Bell et $\mathrm{al}^{3}$ found much room for improvement in communication but no association between a lack of communication and adverse clinical outcomes. A review by Motamedi et $\mathrm{a}^{21}$ on computer-enabled discharge communication showed an improvement in GP satisfaction but no effect on mortality or readmission.

\section{Benchmarks}

Since in Switzerland there are no strictly defined medical regions, many of the GPs were working with different hospitals and therefore a benchmark was given in several comments. Even though the benchmarks could not systematically reflect the situation, the comments showed that working with other hospitals was often less complicated. Many nonuniversity hospitals have obviously already recognized the importance of appropriate communication with GPs. For the university hospital, appropriate communication is even more important, since patients in highly specialized hospitals are frequently severely ill and often experience more internal referrals.

GPs clearly stated that the most important aspect of a good relationship between a hospital and GPs is good cooperation in patient care. Other communications, such as newsletters or even visits from representatives, are pointless if services are not provided.

\section{Strengths and limitations}

Several aspects of cooperation and communication between GPs and a university hospital were observed in this study. The results are consistent with findings in the literature. The authors sought to find specific aspects of cooperation between GPs and the University Hospital Zurich, but, with similar structures in outpatient and inpatient care in the rest of Switzerland and in most countries in Europe, the study results may also be transferred to different settings.

There are limitations to the study: due to the methodological design of a qualitative study and the small number of participants, no representative conclusions can be drawn. The results are also dependent on the interviewer: since group interviews were held, answers could be biased by peer pressure and cannot necessarily be transferred to real life.

\section{Conclusion}

Exchange of information between the hospital and the GP at admission and discharge is essential. At present, involvement of the patient's GP during hospitalization is 
sometimes inadequate. Involvement includes exchanging information after an unexpected clinical procedure and using GPs' information in difficult clinical decisions such as those encountered at the beginning or termination of long-term therapies.

\section{Disclosure}

The authors report no conflicts of interest in this work.

\section{References}

1. Cook RI, Render M, Woods DD. Gaps in the continuity of care and progress on patient safety. BMJ. 2000;320(7237):791-794.

2. Forster AJ, Clark HD, Menard A, et al. Adverse events among medical patients after discharge from hospital. Can Med Assoc J. 2004;170(3): 345-349.

3. Bell CM, Schnipper JL, Auerbach AD, et al. Association of communication between hospital-based physicians and primary care providers with patient outcomes. J Gen Intern Med. 2009;24(3):381-386.

4. Forster AJ, Murff HJ, Peterson JF, Gandhi TK, Bates DW. The incidence and severity of adverse events affecting patients after discharge from the hospital. Ann Intern Med. 2003;138(3):161-167.

5. Bell CM, Bajcar J, Bierman AS, Li P, Mamdani MM, Urbach DR. Potentially unintended discontinuation of long-term medication use after elective surgical procedures. Arch Intern Med. 2006;166(22): 2525-2531.

6. Roy CL, Poon EG, Karson AS, et al. Patient safety concerns arising from test results that return after hospital discharge. Ann Intern Med. 2005; 143(2):121-128.

7. Kripalani S, Jackson AT, Schnipper JL, Coleman EA. Promoting effective transitions of care at hospital discharge: A review of key issues for hospitalists. $J$ Hosp Med. 2007;2(5):314-323.

8. Mesteig M, Helbostad JL, Sletvold O, Rosstad T, Saltvedt I. Unwanted incidents during transition of geriatric patients from hospital to home: a prospective observational study. BMC Health Serv Res. 2010;10:1.

9. Swiss Society of General Internal Medicine. SGIM Charta Zusammenarbeit Spital - Praxis. [The charter of Swiss Society of General Internal Medicine on cooperation between General Practices and Hospitals]. Available from: http://www.sgim.ch/fileadmin/Dokumente/SGIM_Charta_d. pdf. Accessed May 15, 2011. [German.]
10. Snow V, Beck D, Budnitz T, et al. Transitions of Care Consensus Policy Statement American College of Physicians-Society of General Internal Medicine-Society of Hospital Medicine-American Geriatrics SocietyAmerican College of Emergency Physicians-Society of Academic Emergency Medicine. J Gen Intern Med. 2009;24(8):971-976.

11. Development SS. Atlas.ti - The Knowledge Workbench. Version 5.5.5. Information available from: http://www.atlasti.com/.

12. Mayring P. Qualitative Inhaltsanalyse [Qualitative content analysis]. In: Flick U, Kardorff Ev, Steinke I, editors. Qualitative Forschung. Vol 3: Reinbeck bei Hamburg: Rowohlts Enzyklopädie; 2003:468-475 [German.]

13. Swiss Medical Association (FMH). Aerztestatistik 2010 [Physicians' demographics 2010]. Available from: http://aerztestatistik.myfmh2.fmh. ch/ Accessed May 19, 2011. [German.]

14. Rosemann T, Marty F, Bhend H, Wagner J, Brunner L, Zoller M. Utilisation of information technologies in ambulatory care in Switzerland. Swiss Med Wkly. 2010;140:w13088.

15. Lee RG, Garvin T. Moving from information transfer to information exchange in health and health care. Soc Sci Med. 2003;56(3): 449-464.

16. Pantilat SZ, Lindenauer PK, Katz PP, Wachter RM. Primary care physician attitudes regarding communication with hospitalists. Dis Mon. 2002;48(4):218-229.

17. Hruby M, Pantilat SZ, Lo B. How do patients view the role of the primary care physician in inpatient care? Am J Med. 2001;111(9B):21S-25S.

18. Foy R, Hempel S, Rubenstein L, et al. Meta-analysis: effect of interactive communication between collaborating primary care physicians and specialists. Ann Intern Med. 2010;152(4):247-258.

19. Newton J, Eccles M, Hutchinson A. Communication between general-practitioners and consultants - what should their letters contain? BMJ. 1992;304(6830):821-824.

20. Hess DR, Tokarczyk A, O'Malley M, Gavaghan S, Sullivan J, Schmidt $U$. The value of adding a verbal report to written handoffs on early readmission following prolonged respiratory failure. Chest. 2010;138(6):1475-1479.

21. Motamedi SM, Posadas-Calleja J, Straus S, et al. The efficacy of computer-enabled discharge communication interventions: a systematic review. BMJ Qual Saf. 2011;20(5):403-415.
International Journal of General Medicine

\section{Publish your work in this journal}

The International Journal of General Medicine is an international, peer-reviewed open-access journal that focuses on general and internal medicine, pathogenesis, epidemiology, diagnosis, monitoring and treatment protocols. The journal is characterized by the rapid reporting of reviews, original research and clinical studies across all disease areas.

\section{Dovepress}

A key focus is the elucidation of disease processes and management protocols resulting in improved outcomes for the patient.The manuscript management system is completely online and includes a very quick and fair peer-review system. Visit http://www.dovepress.com/ testimonials.php to read real quotes from published authors. 